\title{
ABRAZANDO MI HISTORIA DE VIDA: LA EXPERIENCIA DE VIVIR CON CÁNCER SIENDO ADOLESCENTE
}

\section{HUGGING MY LIFE HISTORY: EXPERIENCING CANCER AS AN ADOLESCENT}

\author{
Martha Orozco Valeta ${ }^{1}$, Luz Enith Maza Padilla², Javier A. Bula Romero ${ }^{3}$ Erimeth Becerra Moreno ${ }^{4}$, Sara Díaz \\ Velásquez ${ }^{5}$
}

Recibido para publicación: Marzo 21 de 2017 - Aprobado para publicación: Abril 25 de 2018

\begin{abstract}
RESUMEN
Objetivo. Explorar la experiencia de vida de un adolescente con cáncer. Materiales y métodos. Estudio cualitativo, con caso único, basado en historia de vida; la técnica utilizada fue la entrevista a profundidad y los resultados se examinaron con base en una versión simplificada de la propuesta de Amedeo Giorgi. Resultados. emergieron dos temas centrales: Diagnóstico: implicaciones para la vida personal, social y familiar de un adolescente con cáncery el apoyo familiar comosoporte indispensable para afrontar la enfermedad. Conclusiones. EI estudio permitió comprender el significado que otorga un adolescente a la experiencia de vivir con osteosarcoma, revelando un mundo distinto y poco explorado. El apoyo de la familia, fue indispensable para la superación del cáncer en el adolescente, podría decirse, que un soporte social sólido en el paciente, favorece los procesos de afrontamiento y adaptación en la experiencia de vivir y sobreponerse a esta condición. En ocasiones el cáncer infantil se visualiza como una enfermedad devastadora, que tiene serias implicaciones para el paciente y su familia, obligándolos a reorganizar su dinámica familiar y social, para adaptarse y luchar contra la enfermedad.
\end{abstract}

Palabras clave: Cáncer, adolescente, historia de vida.

\begin{abstract}
Objective. To explore the life history of an adolescent who experiences cancer. Materials and methods. This is a qualitative study based on single-case life history. In-depth interview was used, and the results were analyzed using a reduced version of Amedeo Giorgi's method. Result. Two central themes emerged from the data: Diagnosis implications for the personal, social and, family life of a teenager suffering from cancer and family support as crucial in coping with the disease. The support of the family was essential in overcoming cancer in an adolescent. Solid social support to the patient favored the processes of living, adapting, coping with and, overcoming cancer. Conclusion. The study allowed the researchers to understand an adolescent's experience of living with osteosarcoma, revealing a particular world that has not been explored in depth. Sometimes childhood cancer is considered a devastating disease, which has severe implications for the patient and his family forcing them to reorganize their family and social dynamics to adapt and fight against that disease.
\end{abstract}

Keywords: Cancer, adolescent, life history.

\footnotetext{
${ }^{1}$ Esp. en Enfermería Médico Quirúrgica, Facultad Ciencias de la Salud, Universidad de Córdoba, Montería, Colombia. Correspondencia: morozcovaleta@correo.unicordoba.edu.co

${ }^{2}$ M.Sc. en Enfermería, Facultad Ciencias de la Salud. Universidad de Córdoba, Montería, Colombia.

${ }^{3}$ M.Sc. en Enfermería, Facultad Ciencias de la Salud. Universidad de Córdoba, Montería, Colombia.

${ }^{4}$ Estudiantes del Programa de Enfermería. Universidad de Córdoba, Colombia.
} 


\section{INTRODUCCIÓN}

Un adolescente con cáncer suele enfrentar un amplio espectro de emociones como el miedo, la ira, la soledad, la depresión, la incertidumbre y la ansiedad (1). Los adolescentes que viven con cáncer están expuestos a sentimientos de culpa y llegar a creer que provocan malestar en su entorno familiar y social; también pueden sentirse indefensos, tristes, irritables, avergonzados por los cambios físicos a los que se enfrenta tras la evolución de su enfermedad, sintiéndose señalados y muchas veces con temor a socializar con sus pares (2), (3).

En consecuencia con lo anterior, poder expresar la experiencia de vida de un adolescente con cáncer, mediante el uso de narrativas, se convierte en un espacio importante para comprender la experiencia de vivir con cáncer; pues se cree que los adolescentes poseen una capacidad especial para la organizar discursos narrativos (4).

En este orden de ideas, poder relatar experiencias de vida a través de narrativas, se convierte en un espacio importante para explorar la experiencia de vida de un adolescente con cáncer.

La historia de vida representa para el adolescente, una herramienta que facilita la comprensión de los acontecimientos significativos de su vida, de manera que pueda afrontarlos con valentía, proyectándose hacia el futuro con más confianza y de una manera positiva (5).

Trabajar con historia de vida es un camino que recorren juntos el investigador y el adolescente, donde pueden existir avances y retrocesos por parte de estos $y$, la tarea principal del investigador es ayudarle a expresar y explorar sus emociones. Una historia de vida es un proceso sensible, que puede plasmarse de diferentes maneras, dependiendo de la creatividad y la imaginación del investigador; lo importante de esta no es el producto final, sino el proceso (5).
La creación de historia de vida de un adolescente con cáncer consiste en: acompañar y ayudar a ordenar, clarificar y comprender los acontecimientos de su vida, partiendo siempre de su propia percepción (6); tratar temas sensibles y utilizar técnicas sencillas para favorecer su comunicación y la expresión de sentimientos (7) y plasmar los recuerdos y experiencias del pasado, las vivencias del presente y las expectativas del futuro (8).

Al explorar las ideas del adolescente es fundamental promover un clima de confianza y aceptación, de manera que éste exprese con la mayor naturalidad posible lo que siente y a partir de ahí, explorar la visión, las ideas y la experiencia de vivir con cáncer. La manera en que el adolescente percibe su propia historia de vida, será siempre el punto de partida.

\section{MATERIALES Y MÉTODOS}

Se trata de un estudio cualitativo descriptivo, con caso único, basado en historia de vida. Según Stake (9), los estudios de casos tienen como características básica, abordar de forma intensiva una unidad, esta puede referirse a una persona, a una familia, un grupo, una organización o una institución.

Existe una amplia discusión en la literatura con respecto a la forma de considerar un estudio de caso; para algunos autores como Muñiz(10), puede ser un enfoque metodológico, mientras que para otros, este tipo de estudio es parte de una estrategia de investigación que se refiere únicamente a un aspecto del método: la selección de la muestra(11).

El estudio de caso como estrategia metodológica, ubica al investigador en un paradigma de investigación cualitativa; el enfoque que se puede adoptar, puede ser diverso, a través de las siguientes estrategias: documentación histórica, historias de vida y estudios de investigación-acción-participación. Para este estudio se utilizó como estrategia metodológica la historia de vida. 
Para presentar los resultados de esta investigación, se tomará como referencia la propuesta de Simons (4), quién indica que el informe final de los estudios de casos, con caso único, describen la forma de contar una historia de vida y la experiencia de un caso concreto. El término "historia de vida" puede tener dos sentidos: el primero corresponde a una estructura narrativa en la que el investigador interpreta el caso; y la segunda una forma específica en la que se plasma el informe escrito. Éste autor propone que el informe final se delimiten los alcances y el contenido del relato y se hacen explicitas las fuentes de información, describiendo cómo se obtuvieron, quién y cuándo las reveló, además de indicar el número de anotaciones de campo y observaciones.

Asimismo, señala que hay distintas formas de organizar y presentar los datos, que dependen del propósito del estudio, pero también del estilo que privilegie el investigador. Para esta investigación se utilizó, un informe descriptivo que implica al lector en el relato, sin introducir interpretaciones densas. La historia no tiene un inicio estipulado sino que refleja cómo se van entretejiendo la información como "un collages de distintas voces", mostrando las metáforas que surgen de la entrevista a profundidad, expresadas a través de narrativas breves del protagonista de la historia; también se pueden presentar fotos e imágenes de los distintos sujetos implicados.

La narrativa del informe debe ofrecer al lector la experiencia de "haber estado allí", transportándolo al lugar, situándolo en el contexto y trayendo la voz de los participantes a través de frases de ellos entrelazadas en el relato. No presenta una investigación neutral y objetiva, sino que intenta trasmitir lo complejo y único del caso en su contexto (4).

En este estudio se utilizó la entrevista a profundidad, definida como encuentros cara a cara entre el investigador y el informante de estudio, encuentros que van dirigidos a la comprensión que tiene el informante, respecto a su vida, experiencias o vivencias personales. (9)

Con relación al número de participantes, Stake (9), plantea que la investigación con estudio de casos no es una investigación de muestras, debido a que este tipo de investigación no se enfoca en obtener generalizaciones, sino que promueve la importancia, singularidad y particularidad de la visión que las personas subrayan frente a un fenómeno puntual, en este caso, a una situación concreta, que corresponde a la experiencia de vida de un adolescente con cáncer.

\section{DESCRIPCIÓN DEL COLABORARDOR DEL ESTUDIO DE CASO}

Esta investigación exploró la experiencia de un adolescente con osteosarcoma, el cual es un tumor maligno que tiene la característica de presentarse en el período de la adolescencia, en la mayoría de los casos aparece en extremidades inferiores, es mortal sin tratamiento, limita la movilidad y restringe las actividades cotidianas en quien lo padece (12). Este tumor óseo representa aproximadamente el 2,5\% de todos los cánceres en menores de 15 años y el 4,2\% en jóvenes entre 15 a 19 años de edad (13).

El colaborador de este estudio, es un adolescente de 14 años; quien a los 13 años de edad fue diagnosticado con osteosarcoma condroblástico del tercio proximal de la tibia izquierda, inició tratamiento con quimioterapia, posteriormente fue intervenido quirúrgicamente para salvamento de la extremidad comprometida, a la fecha de realización de esta investigación había transcurrido un año desde que fue diagnosticado con cáncer. Actualmente se encuentra en proceso de remisión con controles periódicos.

\section{ANÁLISIS DE LOS DATOS CUALITATIVOS EN EL ESTUDIO DE CASO}

La recolección y el análisis de la información se realizó de manera simultánea; teniendo en cuenta el siguiente procedimiento: 
Transcripción fiel: la recolección de la información obtenida durante las entrevistas realizadas, (incluyendo expresiones no verbales), se trascribieron fielmente, con el fin de evitar datos subjetivos, de manera que mantuvieran la pertinencia y la adecuación de la información y así, realizar una completa y rica descripción del fenómeno de estudio, una vez se alcanzó la etapa de saturación teórica.

\section{Selección de las expresiones significativas del} fenómeno: una vez los datos fueron transcritos fielmente, se realizó un rastreo sistemático de los temas que se repetían. Así mismo, se recurrió a las evidencias disponibles, para la construcción de la trayectoria de vida del adolescente, utilizando la narrativa como medio para documentarla.

Técnica para resaltar las expresiones significativas: cada entrevista fue numerada, indicando el número de la hoja y el número de renglones donde se encontraban las expresiones significativas, estás fueron resaltadas con un mismo color para identificarlas en el texto. Una vez identificadas, se realizó un listado de las mismas para su categorización y posterior interpretación teórica.

El análisis de las entrevistas se realizó con base en la propuesta de Amedeo Georgi (14), como se muestra en el siguiente diagrama:

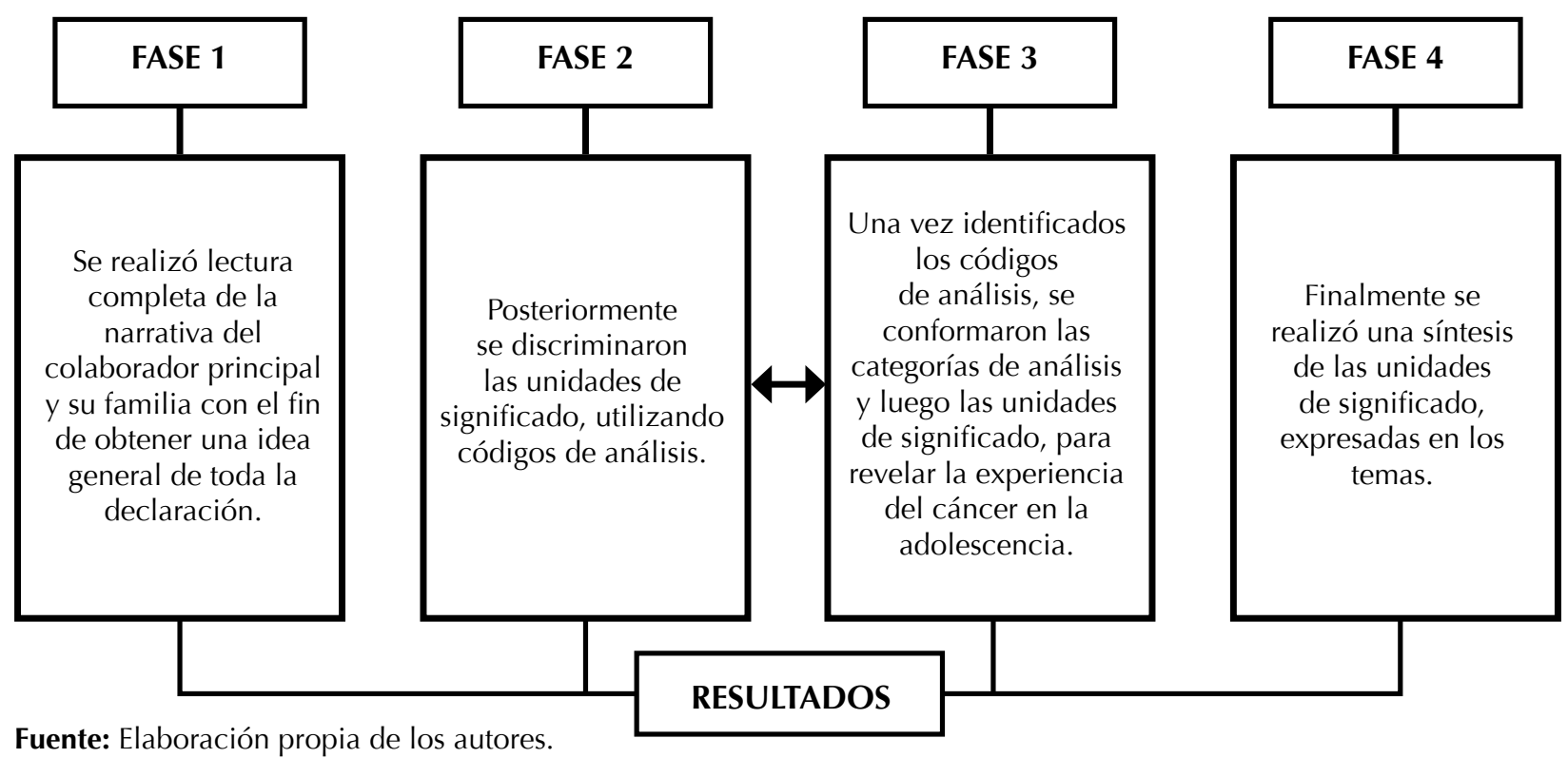

Una unidad de significado se entiende entonces como la característica singular o connotación que se le atribuye a una palabra y que denota una particularidad en el discurso; la unidad de significado busca describir y comprender el fenómeno, más no interpretarlo. La descripción de las vivencias se realizó en función de los temas identificados, los cuales permitieron la construcción de una historia de vida.

La presente investigación pretende ofrecer al lector la experiencia de haber estado allí, transportándolo al lugar, situándolo en el contexto y trayendo la voz de los participantes a través de frases entrelazadas en el relato.

Para presentar los resultados se tomó como referencia, la propuesta de Simons (15), quién indica que el informe final de los estudios de casos, con caso único describe la forma de contar una historia de vida y la experiencia de un caso concreto. 


\section{ORGANIZACIÓN Y PRESENTACIÓN DE LOS RESULTADOS}

Existen distintas formas de organizar y presentar los resultados, que dependen del propósito del estudio, pero también del estilo que privilegie el investigador (16).

A continuación, se describen los temas centrales derivados de la síntesis interpretativa de las unidades de análisis.

Tema 1. Conocer el Diagnóstico: implicaciones para la vida personal, social y familiar de un adolescente con cáncer.

Conocer el diagnóstico de una enfermedad oncológica trae consigo notables implicaciones en la vida personal, social y familiar de un adolescente con cáncer. La primera reacción de un adolescente ante el cáncer puede ser el miedo, es común que este tipo de emoción se presente, porque no se sabe a ciencia cierta qué pasará después.

¡Yo preguntaba que me ocurría y me decían que todavía no estaban seguros de lo que tenía...!

Colaborador

Durante los periodos de hospitalización y tratamiento, convergen una serie de sentimientos como la ansiedad, la desesperación y la incertidumbre; esta situación genera en el adolescente sentimientos encontrados, temor a lo desconocido y en muchas ocasiones confusión (18).

¡Tenía mucha ansiedad, me sentía desesperado y confundido; iera raro lo que sentía!, me sentía mal

y me sentía bien, mal porque estaba ahí, porque me había dado esa enfermedad y bien porque me decía a mí mismo, que podría haber sido peor!

Colaborador

La supervivencia de los pacientes con cáncer, depende en gran medida de la terapéutica médica indicada; la quimioterapia es uno de los tratamientos que más se utiliza en pacientes oncológicos, a pesar de reconocer que esta terapia es necesaria, muchos pacientes, documentan un conjunto de reacciones adversas, que afecta notablemente su calidad de vida (19).

¡Con la quimioterapia no me daban ganas de hacer nada!, pasaba vomitando!... ino comía nada!, a veces demoraba muchos días sin comer, ino podía comer! Colaborador

Los tratamientos oncológicos pueden producir alteraciones significativas en la imagen corporal de un adolescente, de modo que este se tiene que enfrentar a una nueva imagen corporal, por los efectos secundarios del tratamiento. Uno de los cambios más representativos de la quimioterapia, es la pérdida del cabello; en este sentido, Die Trill (20), sostiene que este proceso suele ser más complicado durante la adolescencia; puesto que en esta etapa, la apariencia física tiene especial importancia para un adolescente y convivir con un proceso oncológico complica aún más la situación.

¡Se me cayó el cabello, las cejas y las pestañas, al principio yo me miraba en el espejo y no me reconocía...! Colaborador

Así mismo, el tratamiento interfiere en el desarrollo de actividades que resultaban placenteras para el adolescente, generalmente suele ser el deporte o la actividad física, en la que él esté interesado. En algunos casos el paciente se ve obligado a separarse de su vida social y abandonar sus estudios (21).

¡Ya no puedo hacer deporte de contacto, esto es lo que más duro me ha dado... ipero bueno! hay otras cosas para hacer; como jugar play, ver Nefilx, escuchar música,...!

Colaborador

¡Al principio fue muy duro!... iduro y fastidioso, estar tanto tiempo sin hacer nada!, ipero después ya entendí!. ¡Algunos amigos venían aquí a traerme los trabajos que me dejaban las profesoras!

Colaborador

Un adolescente es reconocido por su vitalidad y su constante necesidad de interactuar con otros 
grupos y mantener vínculos con sus semejantes, pero el tratamiento de la enfermedad, le impide dar continuidad a su anterior estilo de vida, llegando a provocar en el adolescente una sensación de soledad o aislamiento.

¡Anhelaba tanto volver al colegio!, estar con mis amigos, ijugar fútbol... pero todas las semanas me tocaba ir a la clínica por la quimioterapia!...

Colaborador

Uno de los retos más difíciles para las familias con hijos adolescentes con cáncer es preservar la estabilidad familiar (22). La manera como las familias reorganizan su vida depende de factores individuales, familiares, socioeconómicos y culturales. Al respecto Die Trill (20), sostiene que algunas parejas cambian sus patrones de trabajo, de manera que uno de los padres esté siempre con el paciente; otras en cambio, incluyen a los abuelos y otros miembros de la familia en el cuidado del adolescente.

¡Mis abuelos paternos se vinieron a vivir con nosotros más o menos siete meses, por mi enfermedad, fueron un apoyo incondicional!

Colaborador

Por otra parte, un cambio importante observado en la dinámica familiar de estos adolescentes está ligado al vínculo afectivo con la familia (20). Conocer el diagnóstico logró que la familia se uniera más y mantuvieran una comunicación más abierta; estos aspectos fueron de vital importancia en el proceso de adaptación familiar, brindando la oportunidad para que tanto padres como hermanos y abuelos participaran en el cuidado del paciente.

TEMA2. El apoyo familiar como soporte indispensable para afrontar la enfermedad.

Para la síntesis interpretativa de esta unidad de significado y la construcción del tema dos, se tuvo en cuenta las metáforas del grupo familiar primario (padre, madre y hermana) del colaborador de esta investigación.

En el caso de esta investigación, la familia aparece como la primera referencia que se tiene al momento de afrontar la vivencia y el proceso de la enfermedad, en la cual, el paciente y su familia van tejiendo una serie de significados que se ven reflejados en la manera cómo afrontan la enfermedad. Podría decirse que el cáncer no se vive desde la esfera de lo individual, si no que esta enfermedad afecta todo el grupo familiar, por lo tanto, quienes enfrentan y viven de manera más profunda y compleja todo este proceso es el paciente y las personas que lo rodean, a quienes la vivencia de la enfermedad también los impacta de manera significativa (23).

¡Esa experiencia no se la deseo a nadie! ¡Como padre uno nunca se imagina una enfermedad de esta en un hijo!, ja uno inmediatamente, se le viene a la mente que su hijo va a morir! ¡Ese momento es bastante duro!...

Padre del Colaborador

¡Cuando te dan un diagnóstico como este, prácticamente se te derrumba el mundo, sentí un dolor inmenso en mi corazón!..., ime dio rabia, frustración, y me pregunté!... ¿por qué un joven tiene que padecer esta enfermedad?... jel temor de una recaída siempre ha estado, muchas veces pensé que iba a perder a mi hijo!...

Padre del Colaborador

La imagen materna apareció como una figura de soporte emocional. La aceptación y protección familiar también fue de gran ayuda durante todo el tiempo de lucha contra la enfermedad, desde el diagnóstico hasta la declaración de su proceso de remisión.

¡Mi mamá siempre está a mi lado sin importar lo que pase!... ¡Ella fue un gran apoyo para mí!...

Colaborador

¡Mi mamá es más sensible que mi papá!.... ¡La relación con ellos se fortaleció más, todos la pasábamos jugando!.... iPero mi papá ponía el empeño para que yo tuviera todo a tiempo y siempre estaba ahí; el me daba fuerzas hasta para bañarme!... Colaborador 
En la siguiente narración se puede apreciar el apoyo de uno sus padres durante el tiempo de hospitalización.

¡Yo salía de mi trabajo, buscaba la comida y me regresaba, me llevaba la comida y la ropa y allá me cambiaba!..., iuno en la clínica nunca duerme bien, uno iba y trasnochaba y al otro día a trabajar!...

Padre del Colaborador

Por otro lado, se encuentra las reacciones de los hermanos(as) del paciente oncológico, estas pueden ser muy diversas y su expresión varía de acuerdo a la relación que existe entre hermanos, como también del grado de madurez cognitiva y emocional que estos tengan (24).

¡Antes nosotros peleábamos demasiado por cualquier cosa!..., ipero ahora nos contamos más las cosas, ahora nos tenemos más confianza!... Hermana del Colaborador

¡Sentí tristeza porque nunca pensé que le fuera a dar eso!... ;Cuando me dijeron lo que tenía...! ¡bajé mi rendimiento en el colegio!...; ¡me sentía medio sola y triste, porque nunca pensé que a él le fuera a dar eso!.... iMe dio duro ver a mi mamá!..., ella lloraba mucho!...

Hermana del Colaborador

¡Cuando veía a mi hermano se me salían las lágrimas!..., ime dio duro que se fuera porque yo paso con el aquí así peleemos mucho!..., jverlo de esa manera como estaba de flaco!..., ise le caía el pelo!..., ique le hacían esas quimioterapias y eso lo ponía mal!..., isentí miedo que fuera a morir!... Hermana del Colaborador

\section{DISCUSIÓN}

La reacción ante el diagnóstico de cáncer en los adolescentes puede llegar a ser confusa y devastadora; adaptarse a los efectos secundarios del tratamiento puede transformarse en un camino difícil. Según Domínguez y Mendoza, en este contexto es donde la familia fortalece su rol en la recuperación del paciente, brindando soporte y ayuda emocional necesaria para poder afrontar la enfermedad (25).
El apoyo de la familia, la escuela y los amigos fueron indispensables para la superación del cáncer en el adolescente, podría decirse, que un soporte social sólido en el paciente y la espiritualidad, favorece los procesos de afrontamiento y adaptación en la experiencia de vivir y sobreponerse al cáncer (26).

Dentro de los recursos internos también se encuentra la resiliencia, la cual enmarca la capacidad de los individuos para salir fortalecidos de la adversidad (27). Algunos autores como Olivares y Amorín, afirman que un paciente oncológico es considerado muy resilientes situación que se evidencia en este estudio, donde se pudo percibir la capacidad del adolescente para sobreponerse al cáncer y narrar su historia de vida (28).

Cuando un adolescente enfrenta la experiencia de padecer cáncer, realiza una reestructuración de su vida y de sus acontecimientos personales, familiares y sociales (29). Interactuar con otros pacientes oncológicos lo hace más consciente de su propio proceso; percibiendo que su experiencia sirve de enseñanza a las vivencias de otros (30).

\section{CONCLUSIONES}

El impacto del cáncer en un adolescente no solo afecta su vida personal, sino también su entorno familiar, puesto que todos los miembros de la familia se ven afectados por la enfermedad. Tanto el paciente oncológico como su familia, van tejiendo una serie de experiencias que se ven reflejadas desde el ámbito individual y familiar principalmente, al enfrentar y vivir de manera más profunda y compleja este proceso.

Muchas veces el cáncer infantil se visualiza como una enfermedad devastadora, que tiene serias implicaciones en el paciente y su familia, obligando a reorganizar su dinámica familiar y social, para adaptarse y luchar contra la enfermedad.

Los resultados de este estudio permitieron comprender las experiencias de vida de un 
adolescente con cáncer, revelando un mundo distinto y poco explorado.

Para este colaborador, vivir y superar el cáncer fue una experiencia transformadora que permitió en él, asumir las secuelas de un osteosarcoma, redefiniendo y direccionando sus metas y proyectos de vida.

Por otra parte, la historia de vida del colaborador, más que un método, fue un reto que implicó que los investigadores se despojaran de sus propias percepciones, poniendo atención a los hechos que se estaban narrando. Esta experiencia investigativa en particular, muestra al colaborador como el protagonista de una historia de vida con un matiz resiliente.

La necesidad de reflexionar sobre lo que las personas sienten y viven con relación al cáncer, es fundamental para que las enfermeras y enfermeros del área de oncología pediátrica puedan generar estrategias de cuidado, a partir del uso de las narrativas como punto de partida para formular intervenciones efectivas y acordes a las necesidades reales de este tipo de pacientes.

\section{CONFLICTO DE INTERÉS}

Los autores declaran no tener conflicto de interés.

\section{REFERENCIAS}

1. Cicogna et al. Children and adolescent with cancer: experiences with chemotherapy. En: revista latino-americana de enfermagem. Vol. 18 , no. 5 , p. $864-872$

2. Carreño, Sonia. Efecto del cuidado transicional de enfermería en el cuidador de un niño con cáncer. Universidad nacional de Colombia. Bogotá. 2014.

3. Rubia Jael, et al. Importancia de la comunicación en los cuidados paliativos en oncología pediátrica: un enfoque en la teoría humanística de enfermería [en línea]. http://www.scielo. br/scielo.php?pid=s0104116920130 $00300780 \&$ script $=$ sci_arttext \&tlng $=e s$ [citado en junio de 2013].

4. Simons, H. El estudio de caso: Teoría y práctica. Madrid: Morata. 2011.

5. Rico, Diana. Terapia narrativa y cuentos terapéuticos. En: hacer psicología. Issn1870-5618

6. Vidal, Albert. Historia de vida que es y cómo hacerla. Simbolics, psicoterapia [en línea]. http://www.simbolics.cat/cas/ historia-de-vida-que-es-y-como-hacerla/ [citado el 19 de Julio de 2018].

7. Jiménez, J; Martínez, R y Mata, E. Guía para trabajar la historia de vida con niños y niñas: acogimiento familiar y residencial. Universidad de Sevilla. 2010, p. 17.

8. Martínez, M; Romo, R y Huerta, J. Un mapa para llegar a la vida: estudio de caso de un niño con leucemia mediante arte terapia. En: arteterapia. Vol. 6, p. 151.

9. Jiménez, J; Martínez, R y Mata E. Guía para trabajar la historia de vida con niños y niñas: acogimiento familiar y residencial. Universidad de Sevilla. 2010, p. 17.

10. Stake, Robert. Investigación con estudio de casos. Madrid, España. ed. Morata. 1998.

11. Muñiz, Manuel. Estudios de caso en la investigación cualitativa. En: división de estudios de posgrado universidad autónoma de nuevo León. Facultad de psicología. México. p. 1-8.

12. Cabral Gallo M, Delgadillo Hernández, A y Martínez Curiel E. Vivencia del joven con osteosarcoma: estudio de dos casos. Revista Mexicana de Investigación en Psicología. Vol. 4, No 1, 2012.

13. Suárez, Amaranto, et al. Resultados del tratamiento de osteosarcoma convencional de alto grado en niños y adolescentes: 
análisis de supervivencia de una cohorte tratada sin metotrexate. En: revista colombiana de cancerología. Vol. 21, no. 2, p. 86-94.

14. Giorgi, A. Phenomenology and psychological research. Pittsburgh, Penn., EUA: Duquense university press. 1985.

15. Simons, $\mathbf{H}$. el estudio de caso: teoría y práctica. Madrid: Morata. 2011.

16. Hernández, K. S. El método historia de vida: alcances y potencialidades. Recuperado el 12-05-2018 en: http:// www.gestiopolis.com/economia/metodode-investigacióncualitativa.htm

17. Jones, G. R. (1983): Life History Methodology. En G. Morgan (Ed.), Beyond Methods. California: Sage.

18. Montoya, María, et al. Experiencias de niños con leucemia, pertenecientes a la fundación "niño leucémico" de la ciudad de popayán. vol. 7. Enero-diciembre 2013. ISSN: 2011-7191.

19. González y Zambrano, X. Estresores antecedentes al cáncer infantil. Maracaibo.: Universidad Rafael Urdaneta, 2005.

20. Die Trill. Citado por Cadena, A., Castro, G y Martínez, E. vivencias y significados de adolescentes sobrevivientes al cáncer. Trilogía. Ciencia, tecnología y sociedad, 10, 145-163.

21. DieTrill, M. y Vecino, S. El superviviente de Cáncer. En Die Trill, M. Psico-oncología. Madrid, España: ADES ediciones.

22. González, María. El Cáncer Durante La Infancia: Experiencias y Necesidades. Granada, España: UNIVERSIDAD DE GRANADA, 2007.
23. Roca, M. Psicología y cáncer: el proceso de afrontamiento en enfermedades oncológicas malignas en niños, adolescentes y sus familiares. Revista cubana de Salud Pública 37(2)

24. Castro, G. y Franklin, E. Vivencias y significados de adolescentes sobrevivientes al cáncer. Trilogía. Ciencia, tecnología y sociedad, 10, 145-163.

25. Castro, G. y Franklin, E. Vivencias y significados de adolescentes sobrevivientes al cáncer. Trilogía. Ciencia, tecnología y sociedad, 10, 145-163.

26. Domínguez, A y Mendoza, J. Clima social familiar en adolescentes con cáncer y adolescentes con tumores benignos [en línea]. http://sisbib. unmsm.edu.pe/ bvrevistas/ v09_2007/pdf/a07.pdf [citado en Julio 2018].

27. Olivares, G. Resiliencia en pacientes oncológicos, ponencia presentada en II congreso venezolano de psicología, Universidad Metropolitana, caracas, Venezuela.

28. Amorin, k. Ser adolescente con cáncer: un estudio fenomenológico. Investigación presentada psicología sem fronteiras. Sao Paulo, Brasil.

29. González, María. El cáncer durante la infancia: experiencias y necesidades. Granada, España: Universidad de granada, 2007.

30. Ibáñez, Edgar. Beneficio del apoyo psicosocial a la calidad de vida de niños y niñas enfermos de cáncer: una revisión sistemática cualitativa. En: revista colombiana de enfermería. Vol. 4, no. 4, p. 125-145. 